\title{
BUBBLE WARS
}

\section{Sébastien MICHELIN ${ }^{(\dagger)}$ \& Eric LAUGA ${ }^{(\dagger)}$}

${ }^{(\dagger)}$ LadHyX - Département de Mécanique, Ecole Polytechnique - CNRS, France

${ }^{(\ddagger)}$ Department of Applied Mathematics and Theoretical Physics, University of Cambridge, United Kingdom

A microscopic bubble of soluble gas always dissolves in an undersaturated fluid in finite time. This diffusive process is driven by the difference between the gas concentration near the bubble, whose value is governed by the internal capillary pressure through Henry's law, and the concentration in the far field.
The presence of neighbouring bubbles can significantly slow down this process by increasing the effective background concentration and reducing the diffusing flux of dissolved gas experienced by each bubble.
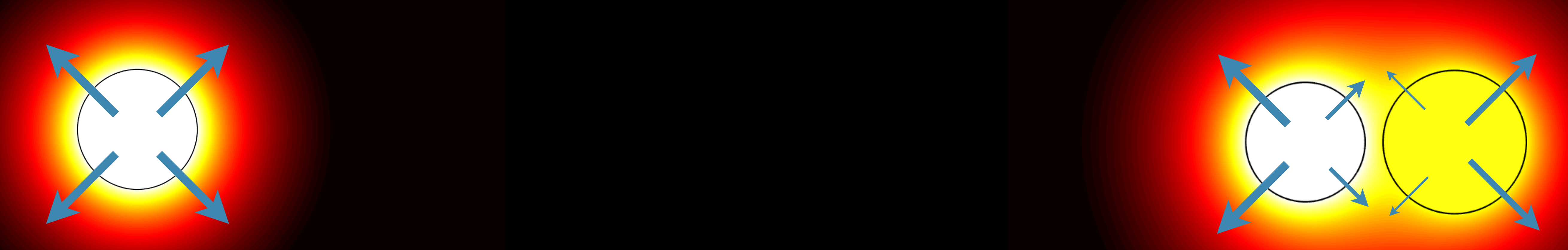

For large lattices of $N$ bubbles, this diffusive shielding effect leads to severalfold increases in dissolution time (scaling respectively as $\log (N), N^{I / 2}$ and $N^{2 / 3}$ for 1D, 2D and 3D regular lattices). For large and/or dense lattices, transient patterns exhibit a complex and highly sensitive dependence on the initial geometric arrangement and sizes of the bubbles.

Below we employ numerical simulations with small fluctuations in initial conditions (size and location of the bubbles) to illustrate this sensitivity and the resulting Bubble Wars where each bubble fights for its diffusive survival.
